\title{
Early-onset autosomal recessive cerebellar ataxia associated with retinal dystrophy: new human hotfoot phenotype caused by homozygous GRID2 deletion
}

\author{
Kristof Van Schil, MSc${ }^{1}$, Françoise Meire, MD, PhD², Marcus Karlstetter, $\mathrm{PhD}^{3}$, Miriam Bauwens, MSc', \\ Hannah Verdin, MSc${ }^{1}$, Frauke Coppieters, PhD ${ }^{1}$, Eva Scheiffert, BSc ${ }^{3}$, Christian Van Nechel, MD, PhD \\ Thomas Langmann, $\mathrm{PhD}^{3}$, Nicolas Deconinck, $\mathrm{MD}, \mathrm{PhD}^{5}$ and Elfride De Baere, $\mathrm{MD}, \mathrm{PhD}^{1}$
}

\begin{abstract}
Purpose: The aim of this study was to identify the genetic cause of early-onset autosomal recessive cerebellar ataxia associated with retinal dystrophy in a consanguineous family.

Methods: An affected 6-month-old child underwent neurological and ophthalmological examinations. Genetic analyses included homozygosity mapping, copy number analysis, conventional polymerase chain reaction, Sanger sequencing, quantitative polymerase chain reaction, and whole-exome sequencing. Expression analysis of GRID2 was performed by quantitative polymerase chain reaction and immunohistochemistry.
\end{abstract}

Results: A homozygous deletion of exon 2 of GRID2 (p.Gly30_Glu$81 \mathrm{del})$ was identified in the proband. GRID2 encodes an ionotropic glutamate receptor known to be selectively expressed in cerebellar Purkinje cells. Here, we demonstrated GRID2 expression in human adult retina and retinal pigment epithelium. In addition, Grid2 expression was demonstrated in different stages of murine retinal development. GRID2 immunostaining was shown in murine and human retina. Whole-exome sequencing in the proband did not provide arguments for other disease-causing mutations, supporting the idea that the phenotype observed represents a single clinical entity.

Conclusion: We identified GRID2 as an underlying disease gene of early-onset autosomal recessive cerebellar ataxia with retinal dystrophy, expanding the clinical spectrum of GRID2 deletion mutants. We demonstrated for the first time GRID2 expression and localization in human and murine retina, providing evidence for a novel functional role of GRID2 in the retina.

Genet Med advance online publication 14 August 2014

Key Words: autosomal recessive cerebellar ataxia; glutamate receptor; GRID2; hotfoot; retinal dystrophy

\section{INTRODUCTION}

Autosomal recessive cerebellar ataxias (ARCAs) are a group of neurodegenerative disorders characterized by a tremendous clinical and genetic heterogeneity. ${ }^{1,2}$ Early-onset ARCAs represent a subgroup for which several disease genes have been identified as playing a role in processes such as DNA damage response, DNA repair, DNA transcription, DNA replication, RNA processing, protein folding and modification, and neurotransmitter metabolism, of which glutamate is an example. ${ }^{1}$

Glutamate receptors were recently implicated in two subforms of early-onset ARCA. First, mutations in GRM1, encoding the metabotropic glutamate receptor 1 , were found in patients of Roma origin with ARCA, characterized by developmental delay, intellectual disability, and a small brain. ${ }^{3}$

Second, a homozygous partial deletion of GRID2, encoding the ionotropic glutamate receptor delta 2 , was found in a consanguineous Turkish family with early-onset ARCA, nystagmus, hypotonia with developmental delay in gross motor skills, encephalopathy with cerebellar ataxia, oculomotor apraxia, and pyramidal tract involvement. ${ }^{4}$ Another recent study reported additional partial biallelic GRID2 deletions in patients with early-onset ARCA, eye movement abnormalities, delayed speech, and intellectual disability. ${ }^{5}$ In addition, a heterozygous deletion of exon 1 and the upstream region of GRID2 was found in an adult with spastic paraplegia, ataxia, frontotemporal dementia, and lower motor neuron involvement. ${ }^{6}$ Prior evidence implicating GRID2 in early-onset cerebellar ataxia was provided by two studies dealing with autoantibodies against the receptor after acute viral infection in two children. ${ }^{7,8}$

Apart from these few human cases, the link between GRID2 and ARCA was already apparent in natural mutants in mouse. Indeed hotfoot (ho) mice are characterized by cerebellar ataxia and jerky movements of the hind limbs. ${ }^{9}$ The hotfoot locus has been mapped to chromosome $6,{ }^{10,11}$ leading to the identification of the Grid2 gene as the underlying disease gene. ${ }^{12}$ The Grid2 gene is a hotspot for deletions; at least 20 different recessive hotfoot alleles have been described, ${ }^{13}$ most of which are deletions disrupting parts of the coding region of Grid2. ${ }^{14}$ This hypermutability of the Grid 2 locus can probably be ascribed to

\footnotetext{
${ }^{1}$ Center for Medical Genetics, Ghent University and Ghent University Hospital, Ghent, Belgium; ${ }^{2}$ Department of Pediatric Ophthalmology, Queen Fabiola Children's University Hospital, Brussels, Belgium; ${ }^{3}$ Department of Ophthalmology, University of Cologne, Cologne, Germany; ${ }^{4}$ Unite Troubles de l'Equilibré et Vertiges, CHU Brugmann, Brussels, Belgium; ${ }^{5}$ Department of Pediatric Neurology, Queen Fabiola Children's University Hospital, Brussels, Belgium. Correspondence: Elfride De Baere (Elfride.DeBaere@UGent.be)
} 
the large size of the gene (1.4 Mb) and, more specifically, of the second intron.

All hotfoot deletions remove parts of the N-terminal extracellular leucine/isoleucine/valine binding protein-like domain of the protein. This domain is crucial for correct trafficking of the protein out of the endoplasmic reticulum to the surface of Purkinje cells, ${ }^{13,15}$ where it is selectively expressed ${ }^{16}$ and thought to play an important role in the organization of parallel fiberPurkinje cell synapses and associated long-term depression. However, the wild-type protein does not seem to form functional ion channels, nor does it bind to glutamate. ${ }^{15}$

In the reported patients with GRID2-associated ARCA, the first mutation is the counterpart of hotfoot mutant ho8J/ho13J, caused by a homozygous deletion of GRID 2 exons 3 and $4,{ }^{4}$ and the second mutation is compatible with mouse mutant ho15J, caused by a compound heterozygous exon 2 deletion. Two other human deletions, one of exon 4 and the other of exon 1 , do not have mouse counterparts.

Here we report a fifth human deletion mutant, characterized by a homozygous GRID2 deletion and mimicking the mouse ho15J mutant. This was found in a proband of consanguineous origin with early-onset ARCA and retinal dystrophy. Whole-exome sequencing in the proband demonstrated no defects other than the GRID2 deletion. Moreover, we demonstrated GRID2 messenger RNA (mRNA) and protein expression in both murine and human retina, suggesting a novel functional role of this receptor in the retina. Our study expands the expression domain of GRID2 and the clinical spectrum of hotfoot deletion mutants in human, which thus far involved only cerebellar and no retinal phenotypes. Finally, we provided further evidence for evolutionary conservation of a hotfoot fragile site between mice and humans.

\section{Patients}

\section{MATERIALS AND METHODS}

The proband is a child of Moroccan origin with cerebellar ataxia and eye motility disorder and originates from a consanguineous family. Both parents and two healthy siblings participated in this study. Ophthalmological examination was carried out with fundoscopy, assessment of eye motility, and electroretinography (ERG). The child underwent neurological assessment including magnetic resonance imaging. Informed consent was obtained, and research protocols adhered to the tenets of the Declaration of Helsinki.

\section{Molecular characterization of GRID2 deletion}

Identity-by-descent and homozygosity mapping. Identityby-descent and homozygosity mapping was performed using genome-wide single-nucleotide polymorphism chip analysis using the HumanCytoSNP-12 BeadChip platform (Illumina, San Diego, CA). Identity-by-descent regions $(>1 \mathrm{Mb})$ were identified using PLINK software ${ }^{17}$ integrated in arrayCGHbase. ${ }^{18}$ Resulting homozygous regions were ranked according to their length and number of consecutive homozygous single-nucleotide polymorphisms, as described by Coppieters et al. ${ }^{23}$

Copy number variation analysis. Copy number variation analysis in the index patient was performed using the aforementioned single-nucleotide polymorphism chips (log $\mathrm{R}$ ratio $+\mathrm{B}$ allele frequency). Subsequently, quantitative polymerase chain reaction was used to perform segregation analysis of the identified GRID2 deletion in the healthy family members. Primers and conditions can be found in Supplementary Table S2 online; design was done as previously described. ${ }^{19}$ qBasePlus software (Biogazelle, Zwijnaarde, Belgium) was used for data analysis, ${ }^{20}$ and two reference genes were used for normalization of the relative quantities. Two positive controls with known copy numbers were used as a reference to calculate the copy numbers. ${ }^{19}$

Delineation of deletion. Further delineation of the deletion was done by iterative rounds of conventional polymerase chain reaction (for primers and conditions, see Supplementary Table S2 online) in both the $5^{\prime}$ and $3^{\prime}$ breakpoint regions. A junction product was obtained by combining the GRID2del_4Aa_F and GRID2del_14Ea_R primers and was sequenced (BigDye Terminator v3.1 Cycle Sequencing Kit on an ABI 3730XL genetic analyzer; Applied Biosystems, Foster City, CA). Sequences were analyzed with Seqscape software (Applied Biosystems).

Bioinformatics deletion. An extensive bioinformatic analysis has been performed on both breakpoint regions of four different molecularly characterized human GRID2 deletions, as previously described ${ }^{21}$ (see Supplementary Text $\mathbf{S 1}$ online).

Whole-exome sequencing. Whole-exome sequencing was performed in the index patient and one healthy sibling. For exome enrichment, the Nextera Rapid Capture Exome kit (Illumina) was used, followed by paired-end sequencing on a HiSeq 2000 (Illumina) by Aros AB (Aarhus, Denmark). The CLC Genomics Workbench version 6.4 (Aarhus, Denmark) was used to read mapping against the human genome reference sequence (National Center for Biotechnology Information, GRCh37/hg19), removal of duplicate reads after mapping, coverage analysis, and quality-based variant calling, followed by further variant annotation using AlamutHT (Interactive Biosoftware, Rouen, France).

\section{GRID2 expression and localization}

mRNA expression analysis. The following human complementary DNAs were assayed: adult and fetal cerebellum, adult cerebral cortex, adult retina, and adult retinal pigment epithelium (Biochain, Newark, CA). The following mouse tissues were assayed: developmental stages P0 to P60 of the retina. Preparation details of murine complementary DNA can be found in Supplementary Text S1 online. An exon-spanning assay was designed for both human GRID2 and mouse Grid2; primers and conditions 
can be found in Supplementary Table S2 online. qBasePlus software was used for data analysis. ${ }^{20}$ Two reference genes validated by the geNorm experiment have stable expression in all tested human tissues, so intertissue comparisons can be made. For murine tissues, $\beta$-actin served as the reference gene.

Retinal samples of human donors. Retinal samples of human donors were obtained from the Eye Bank of the Center of Ophthalmology, University of Cologne, Germany. After dissection of the anterior segment, the remaining tissue included the posterior pole. Remaining vitreous humor was removed to obtain retinal tissue before further processing.
The research followed the tenets of the Declaration of Helsinki.

Immunohistochemistry. Human and mouse retinal sections were used for immunofluorescence analysis, which was performed as previously described ${ }^{22}$ (see Supplementary Text S1 online).

\section{RESULTS}

\section{Phenotype of the proband}

The 2-year-old patient of Moroccan origin was born to consanguineous married parents. He displayed the following anthropometric measurements at birth: weight P97, height P75, and
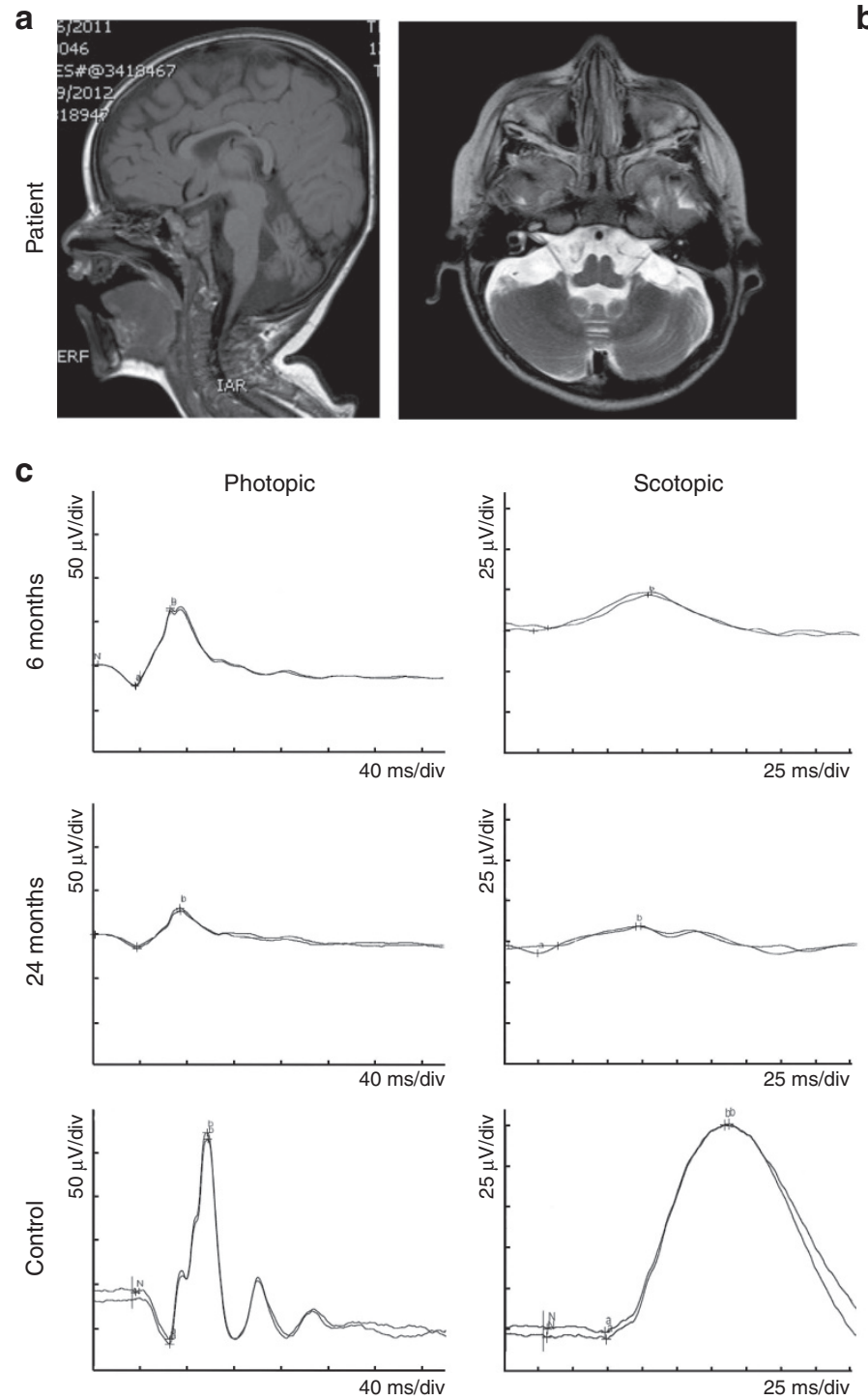

b
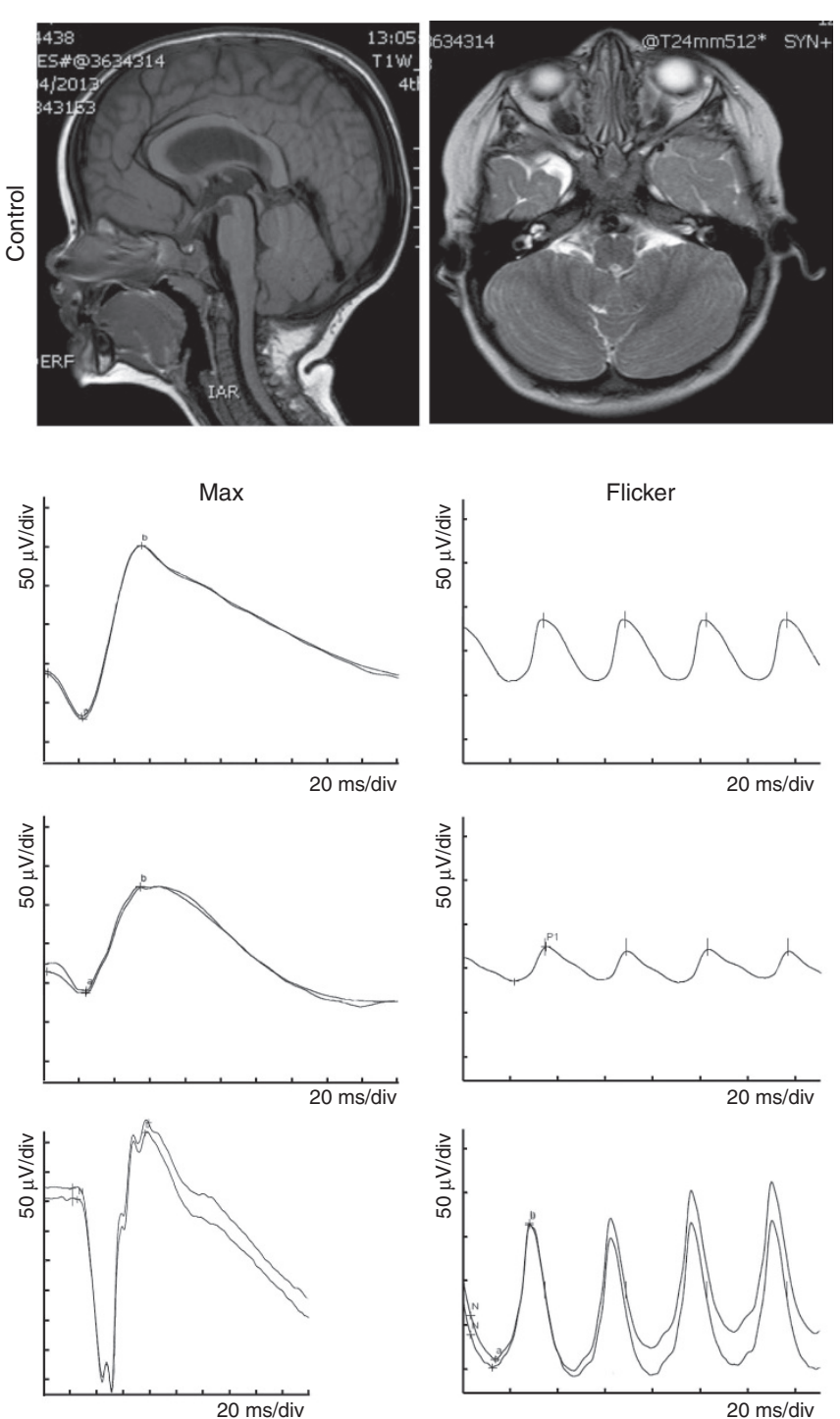

Figure 1 Clinical presentation of the proband. (a,b) Magnetic resonance imaging. (a) (Left) Sagittal T1-weighted axial images of the patient at the age of 1 year showing cerebellar atrophy particularly prominent in the vermis. Supratentorial brain architecture is normal with a complete corpus callosum although appearing slightly thin. (Right) T2-weighted axial image of the patient at the same age, confirming cerebellar atrophy with prominent cerebellar folia also mainly localized in the vermis. (b) Corresponding pictures from control child, 18 months of age. (c) Photopic, scotopic, maximal, and flicker responses on electroretinogram from the affected child compared with responses obtained in the same conditions from a 2-year-old control child. An amplitude deficit can be observed for photopic as well as scotopic responses. At the same scale, the amplitude deficit is obvious between the recordings made at 6 months (upper traces) and 24 months (middle traces) compared with the control subject (lower traces). 
a

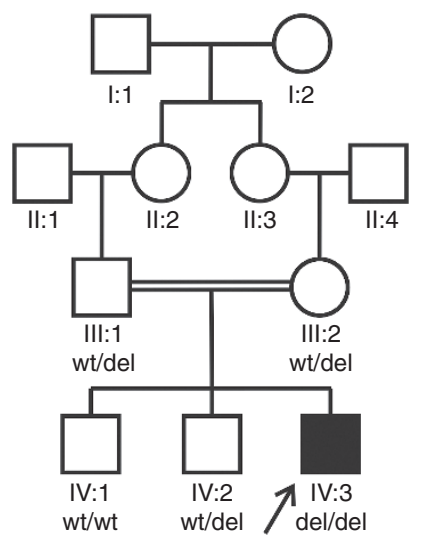

b

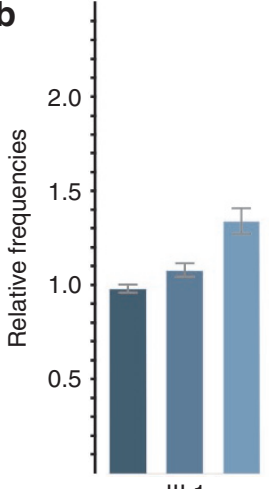

III:1

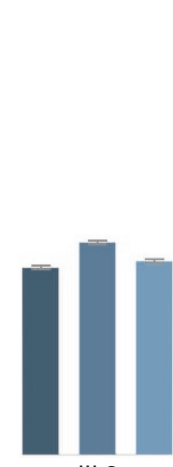

III:2

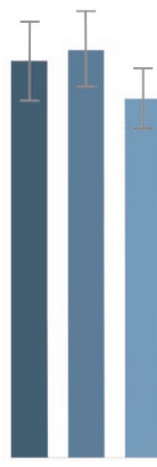

IV:1
GRID2 - intron 1 GRID2 - exon 2 GRID2 - intron 2

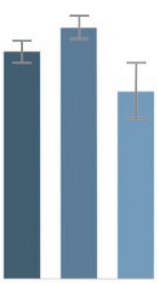

IV:2

IV:3

c

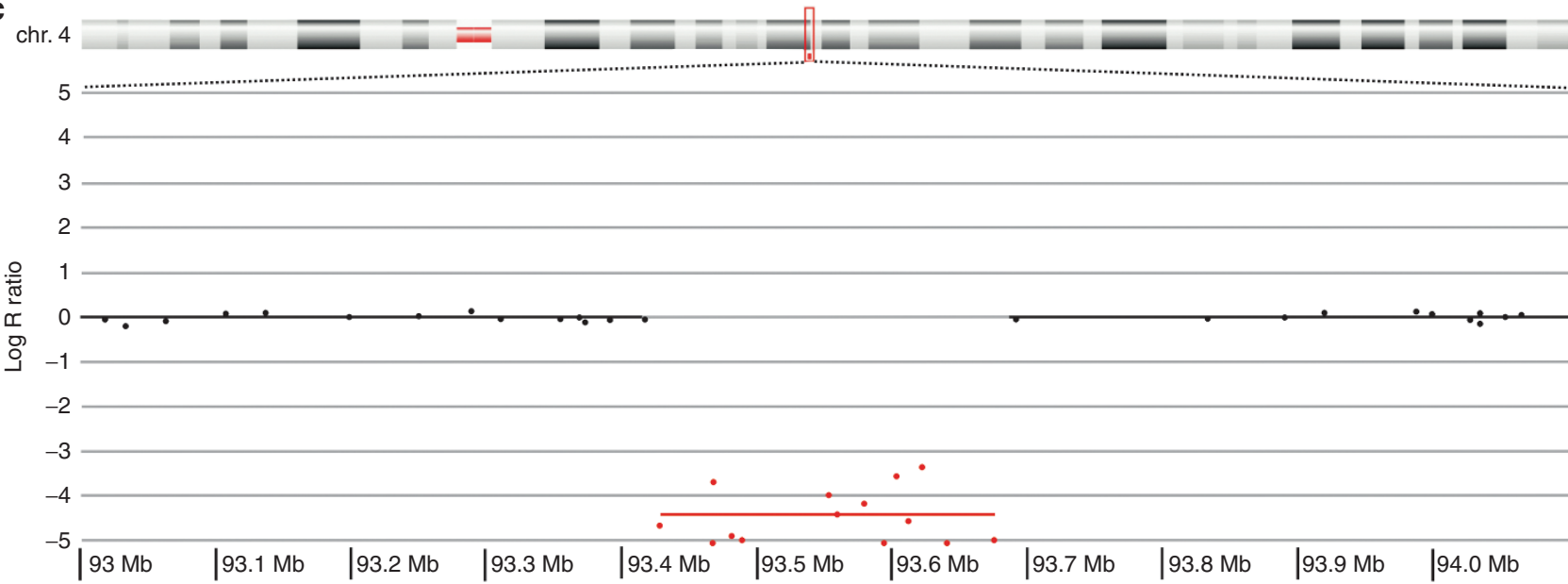

d

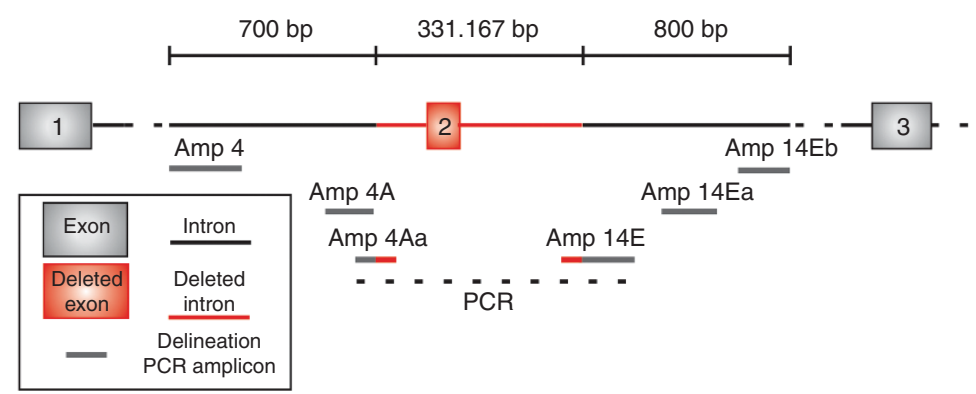

e

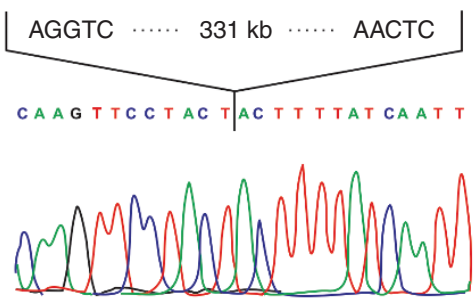

Figure 2 Identification and characterization of GRID2 deletion. (a) Pedigree of the family. Genotypes are indicated for all family members for whom segregation analysis was performed. (b) Segregation analysis of the deletion in all family members. One healthy sibling (IV:1) does not carry the deletion, both parents (III:1 and III:2) and the other healthy sibling (IV:2) carry one copy of the deletion, and the proband (IV:3) has two copies. (c) ArrayCGH profile indicating the presence of a deletion on chromosome 4 (chr.4). (d) Further delineation of the deletion by conventional polymerase chain reaction (PCR). Gray and red boxes and connecting lines indicate respectively nondeleted and deleted exons and introns. Short horizontal lines correspond to designed PCR amplicons and are used to delineate the deletion. The black dotted line indicates the junction product. (e) Sanger sequencing of the junction product led to the delineation of the deletion at nucleotide level, chr4: g.93422866_93754032del. del, allele with deleted exon 2; wt, wild-type allele.

head circumference P90. General physical examination showed pectus excavatum, the presence of some joint laxity, and no significant dysmorphism. On neurological examination, the proband had nystagmus, first recognized at the age of 2 months. Cerebellar system involvement was observed, with truncal hypotonia and ataxic movements of the upper arms. He had abnormal backward head movements especially accompanied by a staring gaze, and his speech was dysarthric. Brain magnetic resonance imaging at 1 year demonstrated cerebellar atrophy particularly prominent in the vermis region (Figure 1). An extensive metabolic workup was normal, as were serum concentrations of albumin, $\alpha$-fetoprotein, creatine kinase, lactate, pyruvate, vitamin E, and cholesterol. Visual evoked potentials, brainstem evoked potentials, electroencephalography, and brain auditory evoked potentials were normal. The neurological course of the disease was rather static, with marked oculomotor 
apraxia and difficulties in fixing gaze. Deep reflexes were always normal, with no sign of pyramidal tract involvement. Slight ankle flexion and inversion contractures were observed.

Ophthalmological examination at the age of 2 months showed visual fixation delay together with a multidirectional nystagmus. An ERG at the age of 6 months showed a severe reduction of both rod and cone responses (Figure 1). At 24 months, all ERG amplitudes, already significantly reduced at 6 months, were further impaired, with an additional average amplitude loss of $\sim 30 \%$ for both scotopic and photopic responses (Figure 1). No fundus abnormalities were observed. Further ophthalmological examination at the age of 24 months revealed a manifest eye movement disorder, mainly tonic upgaze. Both voluntary pursuit and saccadic eye movements were impossible. The child could only observe objects in his peripheral visual fields by turning his head. A nystagmus with small-amplitude beats was best observed when the child attempted fixation (Supplementary Movies S1-S4 online). His visual acuity is estimated to be at least 0.05 .

A summary of the clinical findings of the index case is presented in Supplementary Table S1 online. The oculomotor impairment can be observed in the Supplementary Movies S1-S4 online.

\section{Identification and characterization of homozygous GRID2 deletion}

To identify the underlying genetic cause of the ARCA and Retinal dystrophy (RD) phenotype in the proband, homozygosity mapping combined with copy number variation analysis revealed a homozygous deletion of exon 2 of the GRID2 gene (Figure 2), located on chromosome 4. Breakpoints of the deletion were situated between single-nucleotide polymorphisms rs7684294 and rs7659159 at the $5^{\prime}$ end of the deletion and between rs6852643 and rs1456362 at the $3^{\prime}$ end. Further delineation of the deletion by iterative rounds of conventional polymerase chain reaction led to refinement of the $5^{\prime}$ breakpoint region to $130 \mathrm{bp}$ and $260 \mathrm{bp}$ for the $3^{\prime}$ region. Subsequently, a deletion junction of $650 \mathrm{bp}$ was obtained in the patient but not in the control (Figure 2d). Sanger sequencing of this junction product led to the delineation of the deletion at nucleotide level: chr4:g.93422866_93754032del (Figure 2e). This deletion removes the entire second coding exon of the GRID2 gene, resulting in an in-frame deletion (p.Gly30_Glu81del). Segregation analysis by quantitative polymerase chain reaction confirmed the homozygous deletion in the index patient and demonstrated that both parents and one of the healthy siblings are heterozygous carriers of the deletion, whereas the other sibling is homozygous for the wild-type allele (Figure $2 \mathbf{b}$ ).

To assess the underlying mechanism of the four characterized GRID2 deletions found in our proband and in the ARCA patients reported by Hills et al., ${ }^{5}$ a bioinformatics analysis ${ }^{21}$ was performed on both breakpoint regions of these deletions. A summary of the bioinformatics findings is given in Table 1; visualization of microhomology can be found in Supplementary Figure S1 online. Based on the results of this

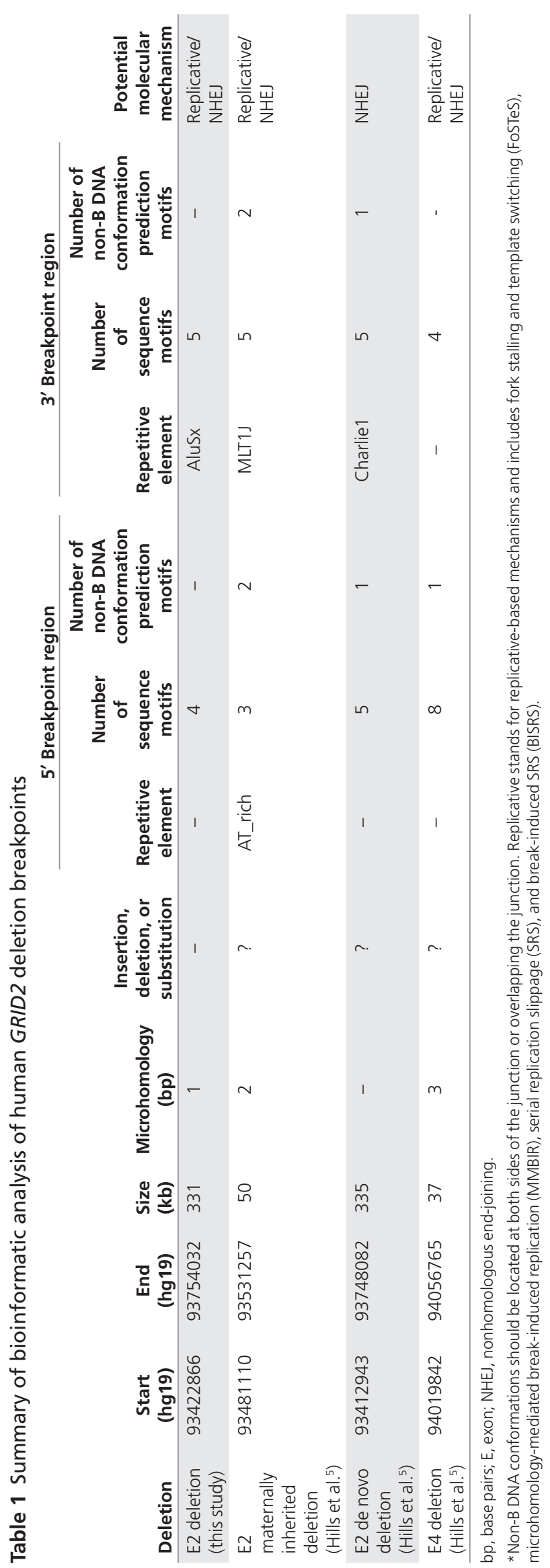




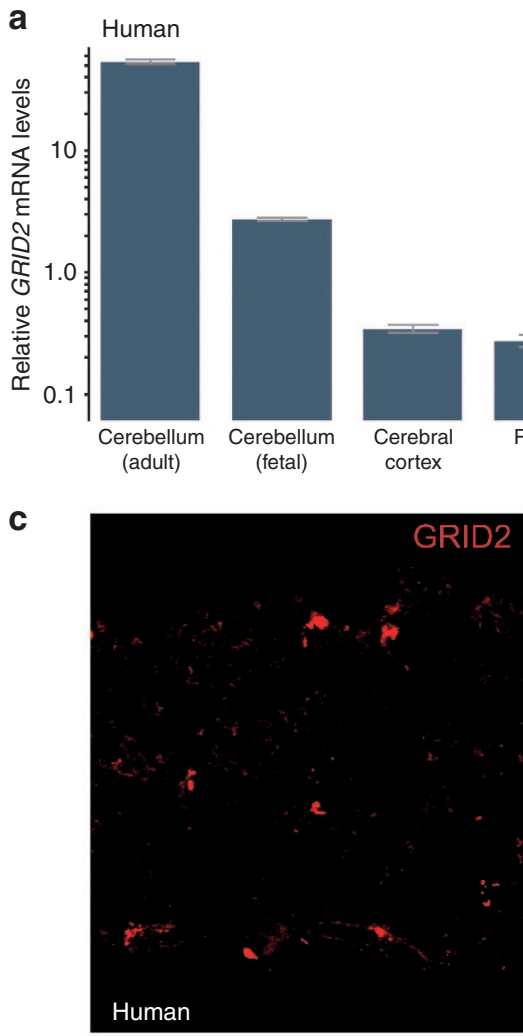

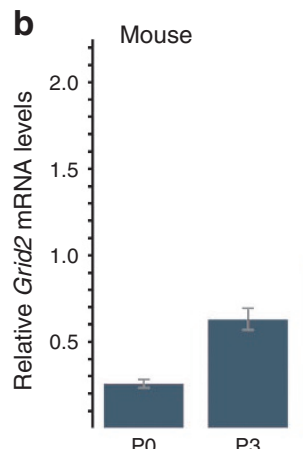

cytes
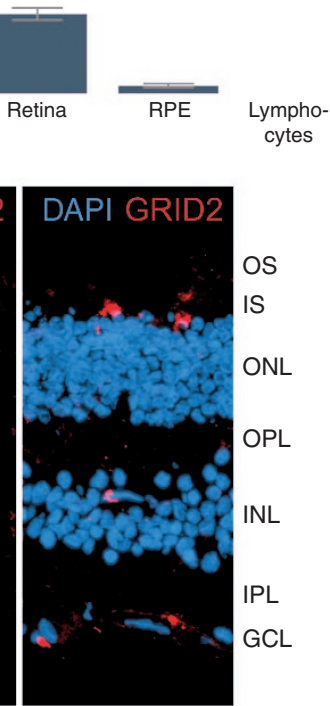

\section{d}
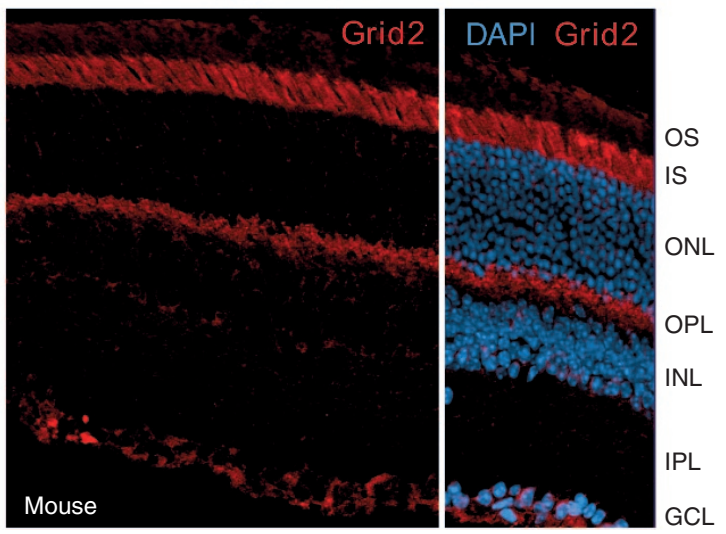

Figure 3 GRID2 expression. (a) GRID2 messenger RNA (mRNA) expression in human complementary DNA (cDNA) of adult and fetal cerebellum, adult cerebral cortex, adult retina, and adult retinal pigment epithelium (RPE), with the strongest expression in cerebellum, similar expression in retina and cerebral cortex, and lower expression in RPE. (b) Grid2 mRNA expression at different developmental stages of retina from neonate (P0) and adult mice (P60). Increasing Grid2 gene expression with progressing development, reaching constant expression levels in adult mice, consistent with gene expression profiles of other retinal genes. (c,d) Representative fluorescent images of horizontal cross-sections of (c) human and (d) mouse retina stained with anti-GRID2 antibody (red, 1:250). Retinal counterstaining was performed with 4',6-diamidino-2-phenylindole (DAPI) (blue). (c) GRID2 immunoreactivity is detected in the photoreceptor inner segments (IS), the outer plexiform layer (OPL), and the ganglion cell layer (GCL). (d) Grid2 immunoreactivity in the murine retina is more globally distributed along photoreceptor IS, the inner nuclear layer (INL)/inner plexiform layer (IPL) margin, and the GCL. ONL, outer nuclear layer; OS, outer segments.

extensive analysis, the studied GRID2 deletions with microhomology may be caused by nonhomologous end-joining $(<5$ bp microhomology) or a replicative-based repair mechanism, favoring the latter because an information scar, typical of nonhomologous end-joining, was not present at the junction for our deletion and was not reported for the deletions described by Hills et al. ${ }^{5}$ The deletion without microhomology is more likely to be caused by nonhomologous end-joining. The presence of sequence motifs can result in genomic instability and the formation of a deletion.

To date, GRID2 deletions in humans and Grid2 deletions in mice have only been associated with early-onset ARCA and not with retinal involvement. To gain further evidence that the phenotype observed in the proband represents a single clinical entity, whole-exome sequencing was performed in the index patient and one healthy sibling. An overview of the variant filtering, performed as previously described, ${ }^{23}$ is given in Supplementary Table S3 online. Variant filtering in 209 RetNet genes (https://sph.uth.edu/retnet/) resulted in 47 remaining variants with a potential pathogenic effect. Subsequently, assuming the occurrence of a homozygous mutation because of the consanguinity in this family, all variants located in a homozygous region $(>1 \mathrm{Mb})$ were filtered, resulting in 48 variants with a potential pathogenic effect. However, based on identity-by-descent and exome data of the nonaffected siblings, variant allele frequency, minor allele frequency, literature or database searches, and exclusion by Sanger sequencing, none of the retained variants in known RD genes or located in identity-by-descent regions could be linked to an RD phenotype (Supplementary Table S3 online). An overview of these variants can be found in Supplementary Tables S4 and S5 online.

\section{Expression analysis of GRID2 transcript and protein in mice and humans}

In previous mouse and rat studies, Grid2 expression was shown selectively in cerebellar Purkinje cells, more specifically at parallel fiber synapses. The recent study by Hills et al..$^{5}$ demonstrated conservation of this selective expression in the human cerebellum. As a first step toward explaining the RD phenotype observed here, we performed expression analysis in human complementary DNA of adult and fetal cerebellum, adult cerebral cortex, adult retina, and adult retinal pigment epithelium, 


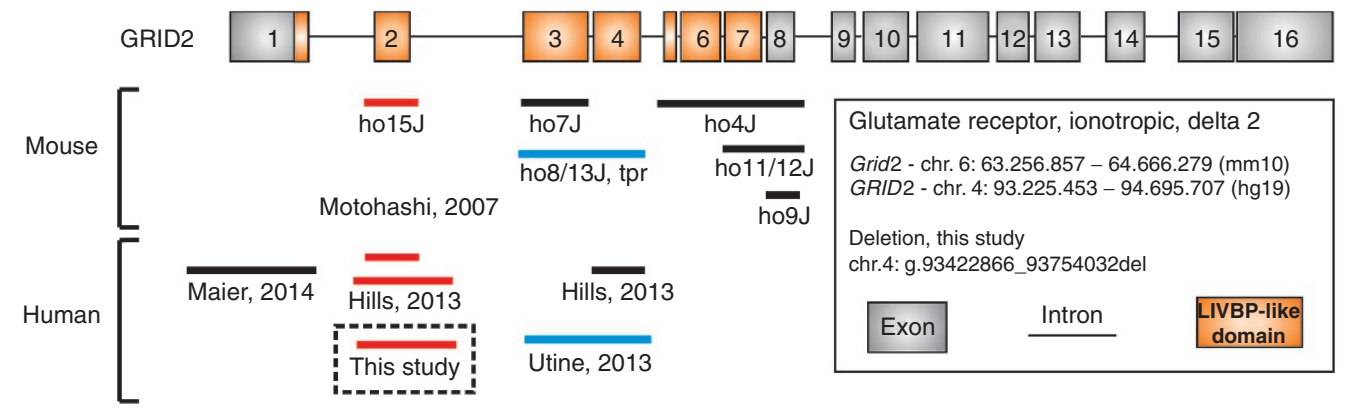

Figure 4 Overview of murine and human hotfoot mutants. Structure of the GRID2 gene; boxes represent exons, connecting lines introns. Colored boxes represent the exons encoding the leucine/isoleucine/valine binding protein-like domain of the protein. Horizontal bars represent GRID2 deletions in mice and humans, depicted in the upper and lower parts, respectively. Corresponding deletions between humans and mice are colored. The red bars represent a homozygous deletion of exon 2, found in ho15J mice, in the patients described by Hills et al. ${ }^{5}$ as a compound heterozygous deletion, and our proband. The blue bars represent a homozygous deletion removing both exons 3 and 4, as described in ho8/13J and tpr mice and the patients reported by Utine et al. ${ }^{4}$ The human deletion of exon 1 is a heterozygous deletion, reported by Maier et al. ${ }^{6}$

demonstrating GRID2 mRNA expression in all of these tissues (Figure 3a). Expression was strongest in cerebellum, followed by similar expression levels in retina and cerebral cortex and lower expression in retinal pigment epithelium. In addition, mRNA expression was assessed at different developmental stages of murine retina, including very young developing retinas from neonatal mice (P0) and adult retinas (P60). Grid2 gene expression increased with progressing development and reached constant expression levels in adult mice, which is consistent with the gene expression profile of other retinal genes (Thomas Langmann and Marcus Karlstetter, personal communication) (Figure $3 b$ ).

Next, we performed immunohistochemistry to investigate GRID2 protein localization in the retina. Our results demonstrate that GRID2 protein is expressed in both the human and murine retina. More specifically, GRID2 localized to photoreceptor inner segments, the outer plexiform layer, and the ganglion cell layer in human and mouse. Faint Grid2 immunoreactivity also was observed at the inner nuclear layer/inner plexiform layer margin in the mouse retina. Grid2 expression in the murine retina was globally distributed, whereas human GRID2 expression was restricted to single cells (Figure 3c,d). A negative control staining without primary antibody can be seen in Supplementary Figure S2 online.

\section{DISCUSSION}

In this study we aimed to identify the underlying genetic cause of early-onset ARCA, eye movement disorder, and RD in a child of consanguineous origin. Our genetic studies revealed a 331$\mathrm{kb}$ homozygous deletion removing the second exon of GRID2, mimicking the deletion in ho15J mice; a recent study showed that dysfunction of cerebellar neuronal circuits underlie the characteristic abnormality of hindlimb movements during locomotion in mice with this deletion. ${ }^{24}$ Involvement of GRID2 mutations in human disease has been reported only recently, when our study was in a final stage. Utine et al. ${ }^{4}$ reported a homozygous deletion of exons 3 and 4 in a consanguineous Turkish family with ARCA and cerebellar atrophy, whereas Hills et al. ${ }^{5}$ described two additional biallelic GRID2 deletions of exon 2 (compound heterozygous) and exon 4 (homozygous) associated with ARCA and eye movements, mainly tonic upgaze. Maier et al. ${ }^{6}$ reported a heterozygous deletion of exon 1 and the upstream region of GRID2 in a 24-year-old man with spastic paraplegia, ataxia, frontotemporal dementia, and lower motor neuron involvement. Four of these six different human deletions have a concordant hotfoot allele in mice (Figure 4), and their associated neurological phenotypes show significant overlap, which is summarized as follows: onset before the age of 1 year, a static neurological course, oculomotor impairment characterized by tonic upgaze, nystagmus, oculomotor dyspraxia, gross motor delay, a less affected mental developmental delay, and progressive cerebellar atrophy on brain magnetic resonance imaging (Supplementary Table S1 and Figure S3 online). Importantly, in our patient with ARCA an early-onset RD was shown, documented by decreased scotopic and photopic responses on ERG. Because this was not documented in the previously reported patients, we cannot completely rule out retinal involvement in these patients. The association of ARCA, cerebellar atrophy, and early-onset RD might point to a GRID2 deletion as an underlying cause, which might prompt high-resolution copy number variation analysis. Likewise, ERG might be a valuable tool in the differential diagnosis of early onset ARCAs with cerebellar atrophy (Supplementary Figure S3 online).

To support the hypothesis that the neurological and retinal phenotypes in the proband are a single entity, we performed whole-exome sequencing, but no obvious disease-causing mutations in known RD or other genes could be found (Supplementary Tables S4 and S5 online). Moreover, expression of GRID2 mRNA could be demonstrated, for the first time, in human adult retina and retinal pigment epithelium and in different developmental stages of murine retina. Similar to other retina-associated genes, low Grid2 mRNA expression was found at early postnatal stages, followed by an increase during development, and reaching a steady state around adulthood. In addition, GRID2 immunostaining was shown both in human and murine retina, localizing to photoreceptor inner segments, 
the outer plexiform layer, and the ganglion cell layer. This pattern is in agreement with previous histological and physiological analyses, indicating that photoreceptor, bipolar, and ganglion cells use glutamate as their neurotransmitter. Many glutamate receptor types are known to be expressed in the retina. To the best of our knowledge, only one of them has been linked with retinal disease when mutated. Indeed, mutations in GRM6, encoding the metabotrope glutamate receptor mGluR6, were found in autosomal recessive congenital night blindness. ${ }^{25}$

Ionotropic glutamate receptors (iGluRs) represent another subtype, directly gating ion channels and mediating rapid synaptic transmission through either kainate/AMPA ( $\alpha$-amino3-hydroxy-5-methyl-4-isoxazolepropionic acid) or $\mathrm{N}$-methyl-D-aspartate receptors. Glutamate binding on iGluRs opens cation channels, depolarizing the postsynaptic cell membrane. Neurons within the OFF pathway (horizontal cells, OFF bipolar cells, amacrine cells, and ganglion cells) express functional iGluRs. ${ }^{26}$ The more restricted expression observed in the human retinal section might be ascribed to the more peripheral, rod-rich location of the section. In addition, this might also suggest a differential expression in rods and cones.

Interestingly, Grid1, the first delta subunit of the iGluRs, was also shown to display sensorial expression, more specifically in the inner ear. ${ }^{27}$ In line with this, mice lacking Grid1 showed impaired hearing. ${ }^{28}$ In addition, Grid $1 \mathrm{mRNA}$ was detected in ganglion and bipolar retinal cells, ${ }^{29}$ reminiscent of the Grid2 immunostaining profile.

One of the drivers of retinal expression of GRID2 might be a cis-regulatory element bound by CRX, a retina-specific transcription factor of which the regulome was characterized recently in mouse retina. ${ }^{30}$ Indeed, two cis-regulatory elements bound by CRX are located in the GRID2 region, and one of them is removed by the homozygous deletion in our proband (Supplementary Figure S4 online). Although we cannot exclude RD and concordant ERG changes in the GRID2 deletion mutants based on the clinical data reported by Utine et al., ${ }^{4}$ Hills et al., ${ }^{5}$ and Maier et al., ${ }^{6}$ the absence of a retinal phenotype in these patients might be explained by intact cis-regulatory elements bound by CRX on at least one of the two alleles.

Of note, the murine ho15J mutant, ${ }^{13}$ which is the counterpart of our human deletion, displays retinal degeneration, which is attributed to its genetic background characterized by homozygosity for the retinal degeneration 1 mutation of the Pde6b gene $(\mathrm{C} 3 \mathrm{HJ}){ }^{5}$ Hills et al. ${ }^{5}$ examined eye movements in both ho15J and Grid 2 knockout mice, both showing larger spontaneous eye movements than their littermates. Therefore retinal phenotyping and immunostaining of the Grid 2 knockout mice, having a C57BL/6 background, might shed more light on the consequences of Grid2 disruption in mouse retina.

With the identification of the new hotfoot mutant in humans, we provide further evidence for evolutionary conservation of a fragile site between mice and humans, which is likely attributed to the large size of the second intron in mice and humans. The hypermutability of this region also is illustrated by the high number of scattered structural variants, especially in the second intron, found in genomic databases such as Ensembl. Despite the large number of GRID2 deletion alleles, their allele frequency is estimated to be very low, taking into account the small number of hotfoot mutants in human reported so far. A bioinformatics study of the breakpoint regions of four different, molecularly characterized deletion alleles in human, including our proband, led to the conclusion that nonhomologous endjoining or a replicative-based repair mechanism are the most plausible mechanisms underlying the deletions, which is in agreement with the scattered location of the breakpoints.

In conclusion, we identified a new human hotfoot mutant associated with retinal involvement. We identified GRID2 as the underlying genetic cause of this entity, thereby providing further evidence for evolutionary conservation of a hotfoot fragile site between mouse and human. Moreover, we demonstrated GRID2 expression in both murine and human retina, providing evidence for a novel functional role of this iGluR in the retina. Our study expands the expression domain of GRID2 and the clinical spectrum of GRID2 hotfoot deletion mutants in humans, which thus far involved only cerebellar and no retinal phenotypes.

\section{SUPPLEMENTARY MATERIAL}

Supplementary material is linked to the online version of the paper at http://www.nature.com/gim

\section{ACKNOWLEDGMENTS}

This work was supported by grants from the Research Foundation Flanders (FWO) (FWO11/KAN/013-31524611; FWO $3 G 079711$ to E.D.B.; FWO/KAN/1520913N to F.C.), Research Fund of Ghent University (BOF10/STA/055 to E.D.B.), the IAP project P7/43 (Belspo), Belgian Medical Genomics Initiative (BeMGI) to E.D.B., and Funds for Research in Ophthalmology (FRO) to K.V.S. K.V.S. is a doctoral fellow from the Institute for Innovation by Science and Technology. F.C. is a postdoctoral fellow, H.V. was a doctoral fellow, M.B. is a doctoral fellow, and E.D.B. is a senior clinical investigator of the FWO. The sponsor or funding organization had no role in the design or conduct of this research.

\section{DISCLOSURE}

The authors declare no conflict of interest.

\section{REFERENCES}

1. Fogel $\mathrm{BL}$, Perlman S. Clinical features and molecular genetics of autosomal recessive cerebellar ataxias. Lancet Neuro/ 2007;6:245-257.

2. Anheim M, Tranchant C, Koenig M. The autosomal recessive cerebellar ataxias. N Engl J Med 2012;366:636-646.

3. Guergueltcheva V, Azmanov DN, Angelicheva D, et al. Autosomal-recessive congenital cerebellar ataxia is caused by mutations in metabotropic glutamate receptor 1. Am J Hum Genet 2012;91:553-564.

4. Utine GE, Haliloglu G, Salanci B, et al. A homozygous deletion in GRID2 causes a human phenotype with cerebellar ataxia and atrophy. J Child Neurol 2013;28:926-932.

5. Hills LB, Masri A, Konno K, et al. Deletions in GRID2 lead to a recessive syndrome of cerebellar ataxia and tonic upgaze in humans. Neurology 2013;81: 1378-1386. 
6. Maier A, Klopocki E, Horn D, et al. De novo partial deletion in GRID2 presenting with complicated spastic paraplegia. Muscle Nerve 2014;49:289-292.

7. Shiihara T, Kato M, Konno A, Takahashi Y, Hayasaka K. Acute cerebellar ataxia and consecutive cerebellitis produced by glutamate receptor delta2 autoantibody. Brain Dev 2007;29:254-256.

8. Shimokaze T, Kato M, Yoshimura Y, Takahashi Y, Hayasaka K. A case of acute cerebellitis accompanied by autoantibodies against glutamate receptor delta2. Brain Dev 2007;29:224-226.

9. Guastavino JM, Sotelo C, Damez-Kinselle I. Hot-foot murine mutation: behavioral effects and neuroanatomical alterations. Brain Res 1990;523: 199-210.

10. Southard JL. Linkage data. Mouse News Lett 1981;64:60.

11. Lalouette A, Christians E, Guénet JL, Vriz S. Construction of a high-resolution genetic map encompassing the hotfoot locus. Mamm Genome 1997;8:903906.

12. Lalouette A, Guénet JL, Vriz S. Hotfoot mouse mutations affect the delta 2 glutamate receptor gene and are allelic to lurcher. Genomics 1998;50: 9-13.

13. Motohashi J, Kakegawa W, Yuzaki M. Ho15J: a new hotfoot allele in a hot spot in the gene encoding the delta2 glutamate receptor. Brain Res 2007;1140: 153-160.

14. Wang Y, Matsuda S, Drews V, Torashima T, Meisler MH, Yuzaki M. A hot spot for hotfoot mutations in the gene encoding the delta2 glutamate receptor. Eur J Neurosci 2003;17:1581-1590.

15. Yuzaki M. The delta2 glutamate receptor: a key molecule controlling synaptic plasticity and structure in Purkinje cells. Cerebellum 2004;3:89-93.

16. Araki K, Meguro H, Kushiya E, Takayama C, Inoue Y, Mishina M. Selective expression of the glutamate receptor channel delta 2 subunit in cerebellar Purkinje cells. Biochem Biophys Res Commun 1993;197:1267-1276.

17. Purcell S, Neale B, Todd-Brown K, et al. PLINK: a tool set for whole-genome association and population-based linkage analyses. Am J Hum Genet 2007;81:559-575.

18. Menten B, Pattyn F, De Preter K, et al. arrayCGHbase: an analysis platform for comparative genomic hybridization microarrays. BMC Bioinformatics 2005;6:124.

19. D'haene B, Vandesompele J, Hellemans J. Accurate and objective copy number profiling using real-time quantitative PCR. Methods 2010;50:262-270.
20. Hellemans J, Mortier G, De Paepe A, Speleman F, Vandesompele J. qBase relative quantification framework and software for management and automated analysis of real-time quantitative PCR data. Genome Biol 2007;8:R19.

21. Verdin $H$, D'haene $B$, Beysen $D$, et al. Microhomology-mediated mechanisms underlie non-recurrent disease-causing microdeletions of the FOXL2 gene or its regulatory domain. PLoS Genet 2013;9:e1003358.

22. Hlawatsch J, Karlstetter M, Aslanidis A, et al. Sterile alpha motif containing 7 (samd7) is a novel crx-regulated transcriptional repressor in the retina. PLOS ONE 2013;8:e60633.

23. Coppieters F, Van Schil K, Bauwens M, et al. Identity-by-descent-guided mutation analysis and exome sequencing in consanguineous families reveals unusual clinical and molecular findings in retinal dystrophy. Genet Med 2014; e-pub ahead of print 13 March 2014 (doi:10.1038/gim.2014.24)..

24. Takeuchi E, Sato Y, Miura E, Yamaura H, Yuzaki M, Yanagihara D. Characteristics of gait ataxia in d2 glutamate receptor mutant mice, ho15J. PLOS ONE 2012;7:e47553.

25. Dryja TP, McGee TL, Berson EL, et al. Night blindness and abnormal cone electroretinogram ON responses in patients with mutations in the GRM6 gene encoding mGluR6. Proc Natl Acad Sci USA 2005;102: 4884-4889.

26. Connaughton V. Glutamate and glutamate receptors in the vertebrate retina. In: Webvision: The Organization of the Retina and Visual System. 2005. http://webvision.med.utah.edu/book/part-v-phototransduction-inrods-and-cones/glutamate-and-glutamate-receptors-in-the-vertebrateretina/. Accessed 6 November 2013.

27. Safieddine $S$, Wenthold RJ. The glutamate receptor subunit delta1 is highly expressed in hair cells of the auditory and vestibular systems. J Neurosci 1997;17:7523-7531.

28. Gao J, Maison SF, Wu X, et al. Orphan glutamate receptor delta1 subunit required for high-frequency hearing. Mol Cell Biol 2007;27: 4500-4512.

29. Jakobs TC, Ben Y, Masland RH. Expression of mRNA for glutamate receptor subunits distinguishes the major classes of retinal neurons, but is less specific for individual cell types. Mol Vis 2007;13:933-948.

30. Corbo JC, Lawrence KA, Karlstetter M, et al. CRX ChIP-seq reveals the cisregulatory architecture of mouse photoreceptors. Genome Res 2010;20: 1512-1525. 